\title{
Consensus molecular subtypes of colorectal cancer are recapitulated in in vitro and in vivo models
}

\author{
Janneke F. Linnekamp ${ }^{1,2}$. Sander R.van Hooff ${ }^{1,2}$ Pramudita R. Prasetyanti ${ }^{1,2} \cdot$ Raju Kandimalla ${ }^{1,2}$ • Joyce Y. Buikhuisen ${ }^{1,2}$. \\ Evelyn Fessler ${ }^{1,2,3}$ - Prashanthi Ramesh ${ }^{1,2} \cdot$ Kelly A.S.T. Lee $^{1,2} \cdot$ Grehor G.W. Bochove $^{1,2}$ - Johan H. de Jong ${ }^{1,2}$. \\ Kate Cameron ${ }^{1,2} \cdot$ Ronald van Leersum ${ }^{1,2}$. Hans M. Rodermond ${ }^{1,2}$ - Marek Franitza ${ }^{4,5}$. Peter Nürnberg ${ }^{4,5}$. \\ Laura R. Mangiapane $^{6} \cdot$ Xin Wang $^{7} \cdot$ Hans Clevers ${ }^{2,8,9} \cdot{\text { Louis Vermeulen } \mathbb{D}^{1} \cdot \text { Giorgio Stassi }^{6} \cdot \text { Jan Paul Medema }}^{1,2}$
}

Received: 23 June 2017 / Revised: 2 October 2017 / Accepted: 9 October 2017 / Published online: 5 January 2018

๑) ADMC Associazione Differenziamento e Morte Cellulare 2018

\begin{abstract}
Colorectal cancer (CRC) is a highly heterogeneous disease both from a molecular and clinical perspective. Several distinct molecular entities, such as microsatellite instability (MSI), have been defined that make up biologically distinct subgroups with their own clinical course. Recent data indicated that CRC can be best segregated into four groups called consensus molecular subtypes (CMS1-4), each of which has a unique biology and gene expression pattern. In order to develop improved, subtype-specific therapies and to gain insight into the molecular wiring and origin of these subtypes, reliable models are needed. This study was designed to determine the heterogeneity and identify the presence of CMSs in a large panel of CRC cell lines, primary cultures and patient-derived xenografts (PDX). We provide a repository encompassing this heterogeneity and moreover describe that a large part of the models can be robustly assigned to one of the four CMSs, independent of the stromal contribution. We subsequently validate our CMS stratification by functional analysis which for instance shows mesenchymal enrichment in CMS4 and metabolic dysregulation in CMS3. Finally, we observe a clear difference in sensitivity to chemotherapy-induced apoptosis, specifically between CMS2 and CMS4. This relates to the in vivo efficacy of chemotherapy, which delays outgrowth of CMS2, but not CMS4 xenografts. Combined our data indicate that molecular subtypes are faithfully modelled in CRC cell cultures and PDXs, representing tumour cell intrinsic and stable features. This repository provides researchers with a platform to study CRC using the existing heterogeneity.
\end{abstract}

Janneke F. Linnekamp and Sander R. van Hooff contributed equally to this work.

Edited by S. Fulda

Electronic supplementary material The online version of this article (https://doi.org/10.1038/s41418-017-0011-5) contains supplementary material, which is available to authorized users.

Jan Paul Medema

j.p.medema@amc.nl

1 Cancer Center Amsterdam, Laboratory of Experimental Oncology and Radiobiology (LEXOR), CEMM, Academic Medical Center, Meibergdreef 9, 1105AZ Amsterdam, The Netherlands

2 Cancer Genomics Netherlands, Utrecht, The Netherlands

3 Present address: Gene Center and Department of Biochemistry Ludwig-Maximilians-Universität München, Feodor-Lynen-Straße 2581377 Munich, Germany

4 Cologne Center for Genomics (CCG), University of Cologne, Cologne, Germany

\section{Introduction}

Colorectal cancer (CRC) is a heterogeneous disease with significant inter-patient differences in prognosis and therapy response. Part of these differences can be explained by the molecular diversity detected in CRC $[1,2]$. Next to the

5 Cologne Excellence Cluster on Cellular Stress Responses in Aging-Associated Diseases (CECAD), University of Cologne, Cologne, Germany

6 Cellular and Molecular Pathophysiology Laboratory, Department of Surgical and Oncological Sciences, University of Palermo, Via del Vespro 131, Palermo 90134, Italy

7 Department of Biomedical Sciences, City University of Hong Kong, Kowloon Tong, Hong Kong

8 Hubrecht Institute, Royal Netherlands Academy of Arts and Sciences (KNAW) and University Medical Centre (UMC), 3584 CT Utrecht, The Netherlands

9 Princess Máxima Centre for Pediatric Oncology, Utrecht 3584 CT, The Netherlands 
classical pathway towards CRC described in the Vogelgram [3], several other pathways, such as the microsatellite instability (MSI) [4] and the serrated pathway [5] have been described. Although these different molecular developmental pathways provide us with an insight into CRC tumourigenesis, it does not fully capture the complexity and heterogeneity of the tumour landscape. In order to provide a more complete insight into this complexity, biologically distinct molecular subtypes based on gene expression were defined and validated [6-11]. Leveraging several original subtyping efforts a consensus was generated resulting in four consensus molecular subtypes (CMS) [12]. CMS1 is enriched for MSI tumours that display immune activation. CMS2 reflects the classical subtype encompassing typical WNT/MYC-driven tumours with epithelial characteristics, whereas CMS3 is enriched for KRAS-mutated tumours with activation of metabolic pathways. Finally, CMS4 has mesenchymal features, shows a high stromal content and activation of TGF- $\beta$ and VEGFR pathways. Besides the biological differences, clear clinical distinctions are evident with poor prognosis for CMS4 and a relatively good prognosis for CMS1 [12].



Fig. 1 Characterising the CRC cell line panel. a This table shows whether the cell lines $(n=43)$ in the panel have mutations present in the five most commonly perturbed CRC pathways. To the right the perturbation frequency is shown for MSI and MSS lines. b Additional cell line properties, including CIMP status and methylation of the two Mir200 loci and three Wnt target genes. Samples were defined as CIMP high or CIMP low when $\geq 6$ or $1-5$ out of 8 CIMP markers were positive, respectively. CIMP negative was defined as $<1$ marker positive. CMS Consensus Molecular Subtype, $C I M P=\mathrm{CpG}$ Island Methylator Phenotype, $M S I=$ Microsatellite instable, $M S S=$ Microsatellite stable 
Traditionally, oxaliplatin and 5-fluorouracil (5-FU) have been the backbones of CRC treatment, but other options have become available including irinotecan, Bevazucimab, EGFR targeting agents and more recently immune-modulatory antibodies [13-15]. With treatment options increasing, patient selection and therapy response prediction is becoming increasingly important to avoid unnecessary side effects and healthcare costs. In this context, cell line panels are valuable tools for investigating new drugs and predictive biomarkers [16,17]. In addition, patient-derived xenografts (PDXs), which are established by implantation of tumour fragments and faithfully model the original tumour in mice, have recently been gaining ground [18-21]. In addition, the development of 3D patient-derived spheroid and organoid cultures has been of great added value as they retain a hierarchy that may better reflect the original crosstalk and signalling present in tumours [22-25].

Recent work revealed that CMS classification can be used to predict therapy response. A subset of CMS2 was shown to display sensitivity towards oxaliplatin, while all other subtypes appear largely resistant [26]. In contrast, in metastatic disease irinotecan appears to selectively affect CMS4-like cancers [27]. The implications of these findings are still limited from a clinical perspective, due to the lack of properly stratified preclinical models mimicking the different CMSs. Although CRC cultures and PDX models have been shown to recapitulate the patient's tumour $[21,28]$, efforts to classify them in distinct CMSs has been unsuccessful so far. Strikingly, recent studies suggested that the stromal component in a tumour is decisive for the mesenchymal transcriptome of CMS4 $[29,30]$. Considering the fact that both primary CRC cultures and PDXs lack a (human) stromal component, this has complicated their consensus molecular subtyping.

In a previous study, we were able to successfully classify cell lines into subtypes [6]. Similarly, Medico and colleagues established subtypes for a large panel of cell lines, but both studies did not employ the CMS classification [31]. In this paper, we characterise a panel of CRC cell cultures, including CRC cell lines, primary cultures and PDX models and stratify them into all four CMSs. In contrast to previous reports [21,29,30], we detected CMS4 faithfully in all model systems, indicating that CMS4 can be defined as a tumour cell-intrinsic phenotype in addition to the observed accumulation of stromal cells. Furthermore, we find that these models recapitulate clinical characteristics of patient cohorts.

\section{Results}

\section{Characterisation of cell lines}

To determine whether CRC is faithfully modelled in cell lines available from commercial sources, a panel of 43 CRC cell lines was collected (Supplementary Fig. S1a). First, the mutational patterns were compiled (Supplementary Fig. S2) and a comprehensive overview was generated of five major pathways altered in CRC (Fig. 1a) [32]. Similar ratios of pathway alterations for Wnt, P53 and RTK/RAS pathways were detected in the cell lines as compared to patients. However, PI3K and TGF- $\beta$ pathway mutations showed significantly lower incidence in cell lines (Fig. 1a). Especially PI3K pathway mutations were scarce in the MSS lines, while TGF- $\beta$ pathway mutations were hardly observed in MSI lines. The established cell lines contain a relatively large fraction of MSI lines compared to the patient population. MSI did associate with CIMP in cell lines, as it does in patients (Fig. 1b). Wnt target methylation, which is associated with prognosis [33], was most often observed in MSI/CIMP-high cell lines. In contrast, miR200 methylation, which is linked with mesenchymal features [34], was associated with MSS/CIMP-low cell lines. Together this points to the fact that CRC cell lines represent a heterogeneous set of models comparable to patient heterogeneity.

\section{Subtyping of CRC cell lines}

CMS classification allows for identification of 4 biologically distinct subgroups in CRC. There is however an ongoing debate about the identity of subtypes within the tumour epithelial compartment, as it is suggested that the mesenchymal features detected in CMS4 are the result of the large stromal component in these cancers $[12,24,29,30]$. Nevertheless, the tumour epithelium is considered to be the driving force behind stromal influx, and is therefore likely also showing specific features. Therefore we determined whether CMS classes can also be identified within cell lines. Different publicly available gene expression datasets of the 43 CRC cell lines were collected and used to employ a number of different classifiers. These include classifiers from literature as well as newly developed cell linespecific classifiers, which use a selection of genes stably expressed across patient tumours and cell lines (Supplementary Fig. S3). In contrast to previous subtyping efforts [24,30], which used direct comparison to tumours, our stratification approach revealed the presence of all 4 CMSs. However, consistency of subtype assignment could vary strongly between classification methods and datasets. Therefore, final CMS label assignment was considered feasible only for cell lines that were consistently stratified ( $>66 \%$ concordance, Supplementary Fig. S4). Ultimately, we confidently stratified 29 of 43 cell lines (Fig. 2). The percentage of cell lines assigned to CMS1-4 (respectively $24 / 41 / 10 / 24 \%$ ) was roughly similar to ratios previously observed in patient sets $(16 / 43 / 15 / 26 \%)$, with an expected overrepresentation of CMS1 due to the higher level of MSI+ lines.

For further characterisation 18 representative lines (4 CMS1, 5 CMS2, 3 CMS3 and 6 CMS4) were selected. 
Fig. 2 Gene expression of the CMS stratified CRC cell line panel.Heatmap showing the gene expression profiles of the cell line panel $(n=43)$, separated by CMS. Genes were selected based on their upregulation in a particular CMS (25 genes per CMS). Genes and samples (within CMS) were clustered using the Pearson correlation coefficient as a distance measure. MSI cell lines are shown in red lettering. Abbreviations: $C M S$ Consensus Molecular Subtype, MSI Microsatellite instable, MSS microsatellite stable
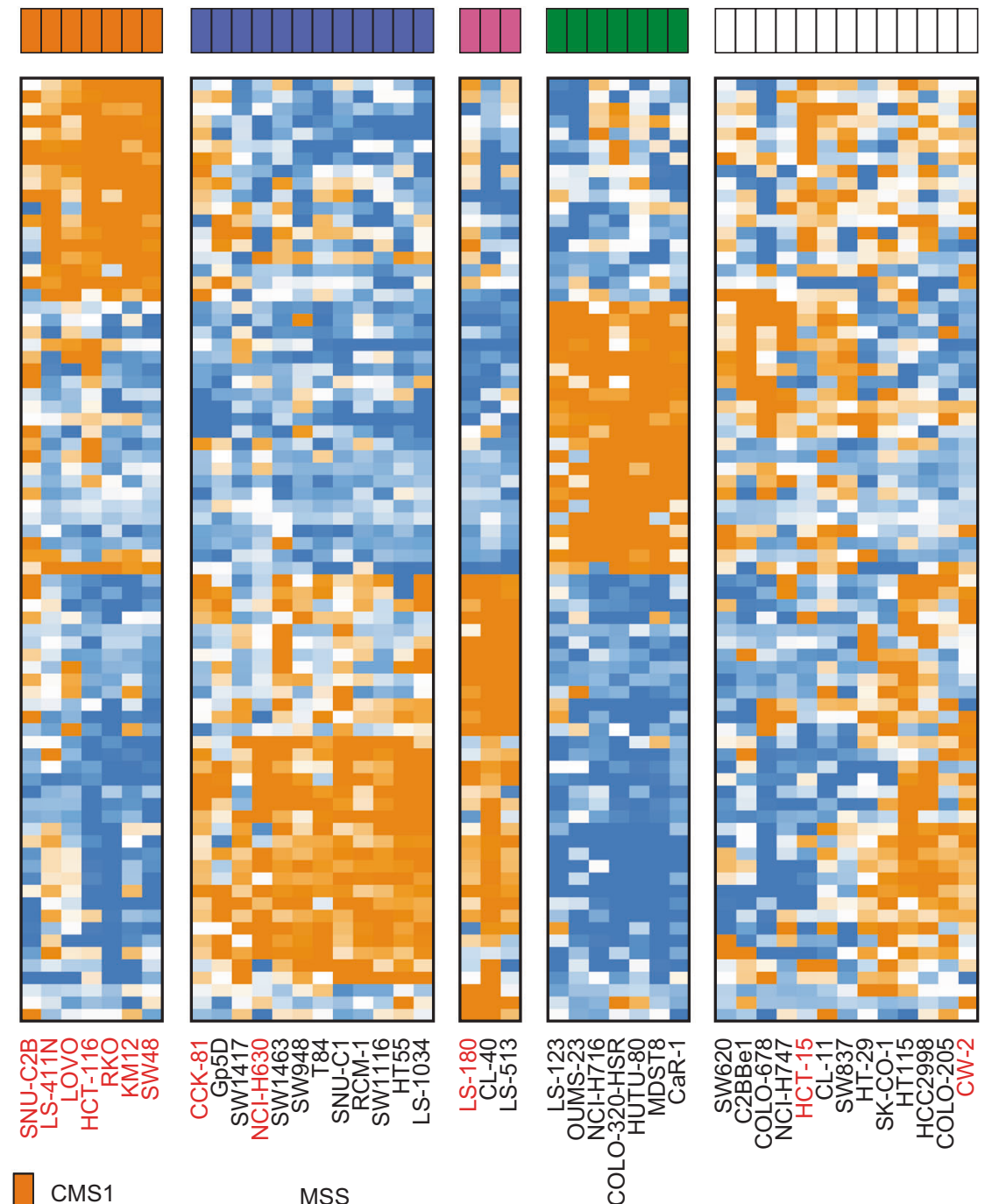

MSS

MSI

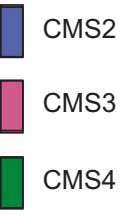

Standardised expression

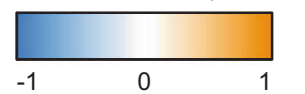

No subtype

First, we confirmed the identity of these cell lines using STR genotyping and a cross-correlation of gene expression profiles (Supplementary Fig. S5). This indicated that CRC cell lines, despite long-term culture and often chromosome instability, display a relatively stable gene-expression profile that can be replicated easily between different laboratories and over longer periods of time and culture.

\section{PDX classifier}

The current data point to the presence of all CMSs in cell lines. However, this subtype assignment is achieved without any prior knowledge of the CMS of the patients from whom these cell lines were derived as this information is not available. To circumvent this caveat a biobank was employed for which gene expression profiles were acquired from colorectal cancers and their respective PDX models. Stratifying the PDX models in this dataset, and especially the mesenchymal CMS4 subtype, represented a specific challenge as currently used CMS4-specific gene expression signatures are connected to stromal accumulation [29,30], which in PDX models is replaced by murine tissue [35] and not or only partially detected by human-specific microarray platforms. Consequently, it has been reported that CMS4 cannot 
Fig. 3 PDX classifier a The PDX classifier is a composite of three consecutive classifiers designed to CMS stratify PDX models using the decision tree shown. The three consecutive classifiers have been designed to work independently gene expression originating from the tumour micro-environment, which is absent from the PDX expression profiles. b The PDX classifier was used to stratify PDX models $(n=34)$ (Passage 1 (P1), Passage 2 (P2) and Passage 3 (P3)) derived from patient tumours $(n=15)$ with known CMS. The results show strong concordance between patient tumours and their associated PDX (Cohen's kappa coefficient $=0.86, p=2.62 \times$ $10^{-12}$ ). c The PDX classifier was also applied to 26 cell lines from the panel. The table compares the results of the PDX classifier with the results of the concordance stratification, showing an almost perfect concordance (Cohen's kappa coefficient $=0.94, p=5.11 \times$ $10^{-15}$ ). Abbreviations: $C M S$ Consensus Molecular Subtype, PDX Patient Derived Xenograft
A



B

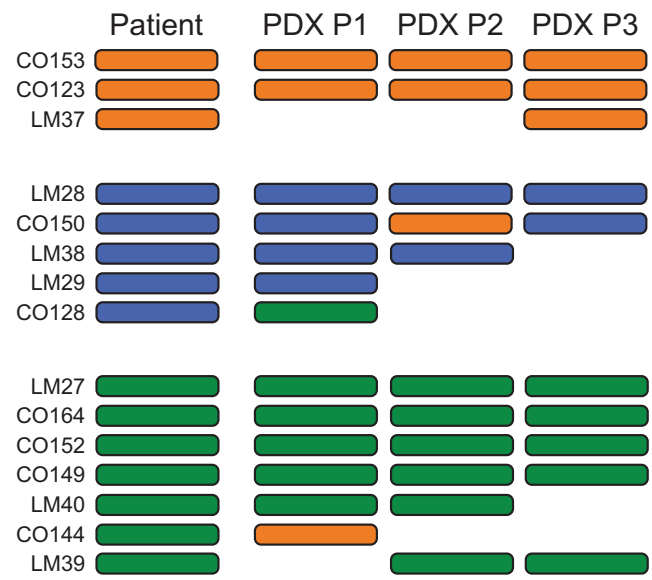

C

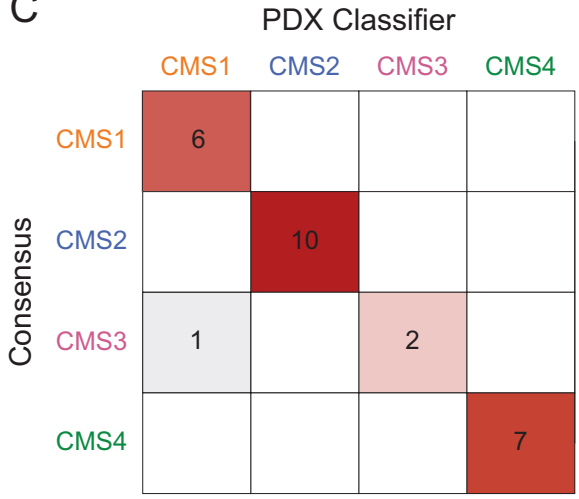

be detected in PDX models [24,30]. We reasoned that CMS4 is, similar to cell lines, present in PDX models and can be identified provided that one zooms in on tumour cell-intrinsic differences between subtypes. As the cell line stratification described above still depends to some extent on genes that are also expressed in stroma, a bioinformatics workflow was designed to develop a CMS classifier that was independent of stromal gene expression. By comparing published datasets of stromal and tumour-epithelial gene expression (detailed in Material and methods) [29,30], a geneset was defined that allowed for stratification based on the expression that is unique to tumour epithelium (Fig. 3a). A subsequent multistep PDX classifier was designed that could faithfully stratify PDX models giving a subtype annotation that in the majority of cases was identical to the patient's CMS (Fig. 3b). This analysis thus refutes the notion that CMS4 is merely a result of stromal accumulation and instead shows that this also constitutes a tumour-intrinsic feature. Moreover, our analysis indicated that most PDX models maintained the original subtype even upon multiple passaging, suggesting that also this represents a stable tumour cell-driven trait.
To determine whether this epithelial-specific PDX classifier could also identify CMSs in cell lines it was used to stratify the CRC panel described above. This revealed a near complete concordance (96\%) with our previous stratification (Fig. 3c). So even when purely epithelial-specific gene expression is used CMS1-4 can be detected in CRC cell lines, providing independent support for the presence of consensus molecular subtypes. Of note, the only cell line that was differentially classified shifted from CMS3 to CMS1, which matched its MSI+ status. We, however, decided to maintain its original CMS3 classification as the majority of classifications pointed towards this subtype.

\section{Characterisation and classification of primary cell cultures}

Having established a panel of CMS-stratified cell lines we decided to use these as a gold standard to classify a set composed of 33 primary cell cultures. Importantly, all primary cultures could reliably be stratified into 4 subtypes (Fig. 4a), although CMS2 cultures were clearly 
Fig. 4 Characterising the primary cultures a Table showing primary culture properties such as MSI, CIMP, methylation of the Mir200 loci and mutations in 5 known CRC driver genes. b Heatmap showing the expression of the CRC cell line panel (only stratified cell lines, $n=29$ ) and the additional primary cultures $(n=33)$, separated by CMS. Genes were selected based on their upregulation in a particular CMS (25 genes per CMS). Genes and samples (within CMS) were clustered using the Pearson correlation coefficient as a distance measure.

Abbreviations: $C M S$ Consensus Molecular Subtype, CIMP CpG island Methylator Phenotype, MSI Microsatellite instable, MSS Microsatellite stable

\section{A \\ cмs -

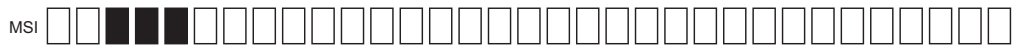



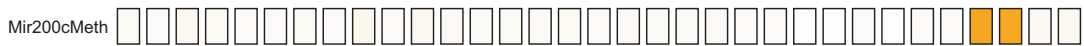 Mir20obameth

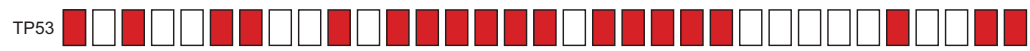



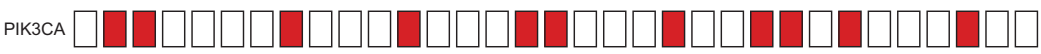 BRAF



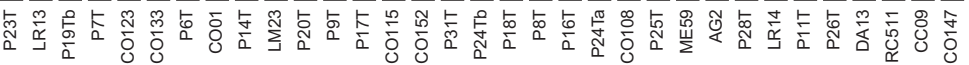

$\begin{array}{lllll}\square & \text { MSI } & \text { CIMP high } \\ \square & \text { CMS2 } & & \\ \text { MSS } & & \text { CIMP low } \\ \square & \text { CMS3 } & \square & \text { CIMP negative } \\ \text { CMS4 } & & \end{array}$

$\mathrm{B}$

CRC cell lines

Hubrecht

Biobank



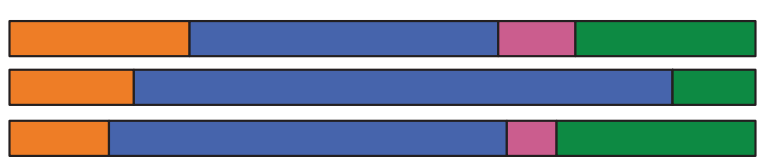

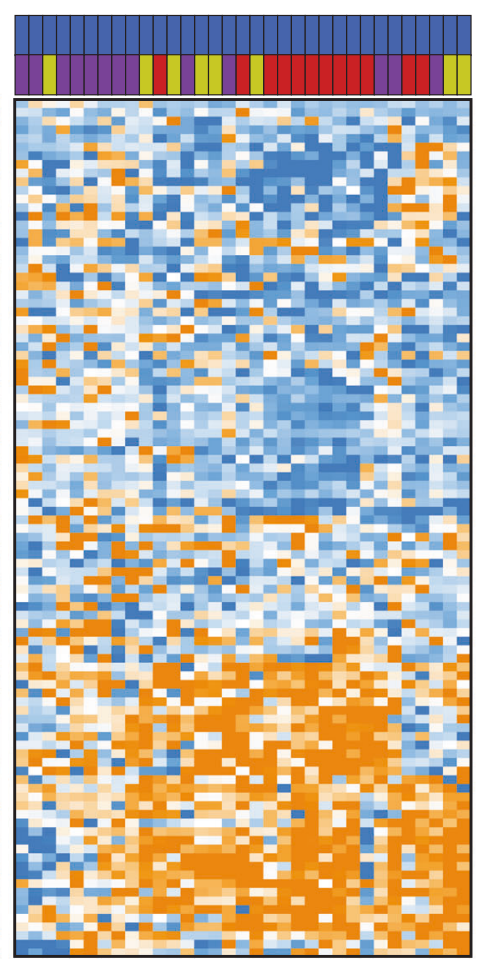

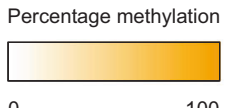

0

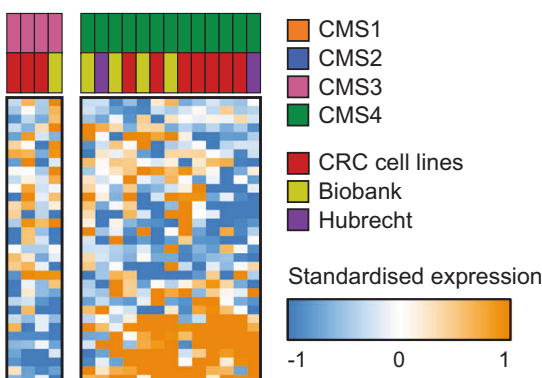

Pathway mutation No pathway mutation

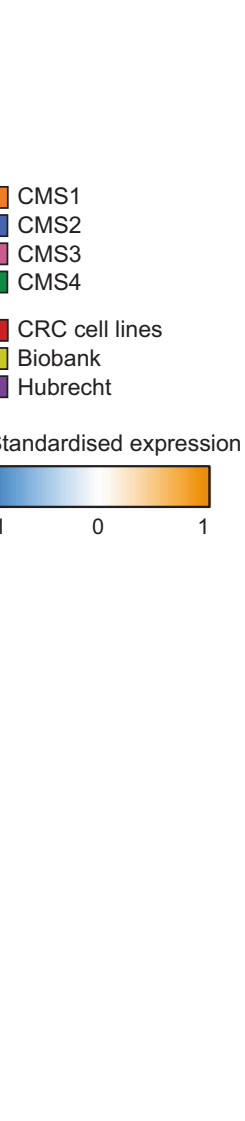


A

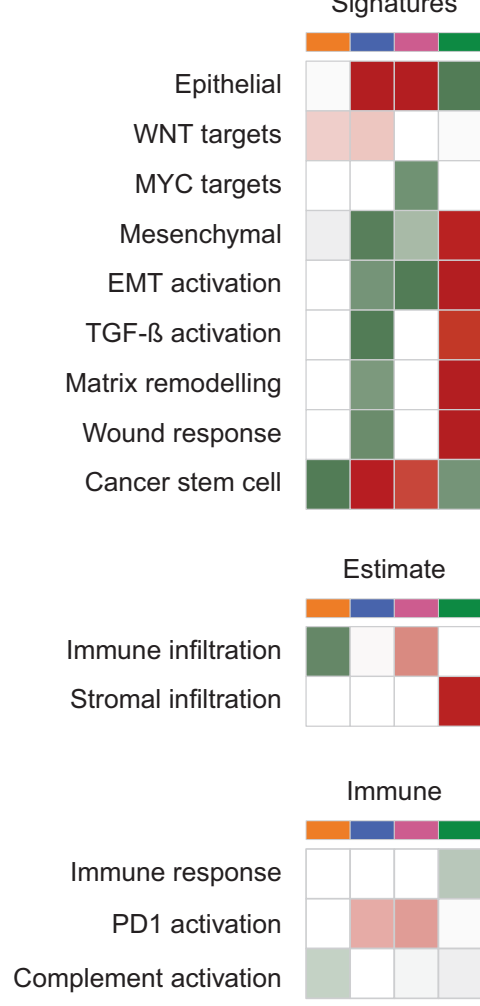

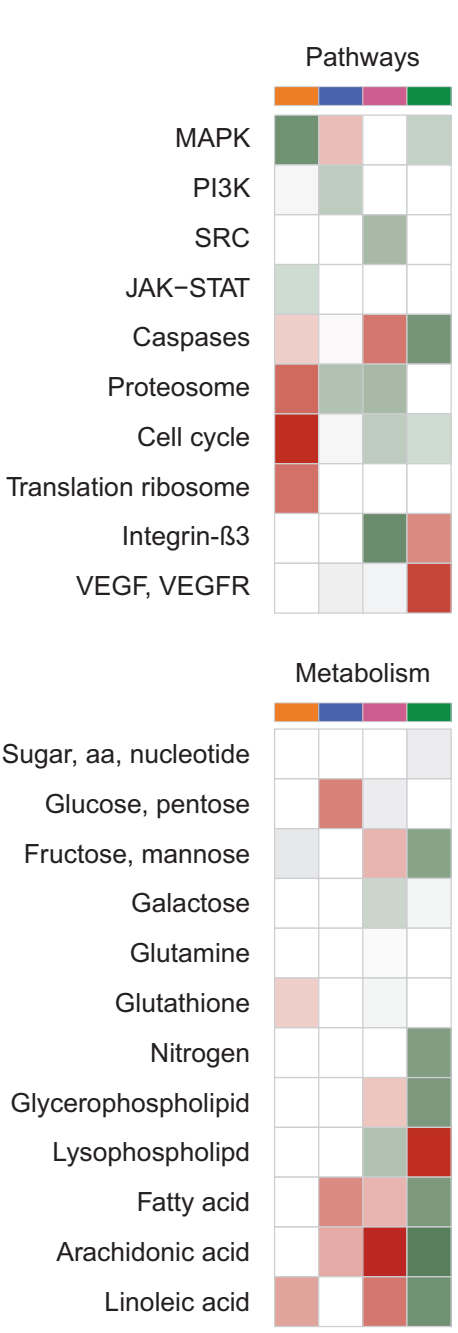
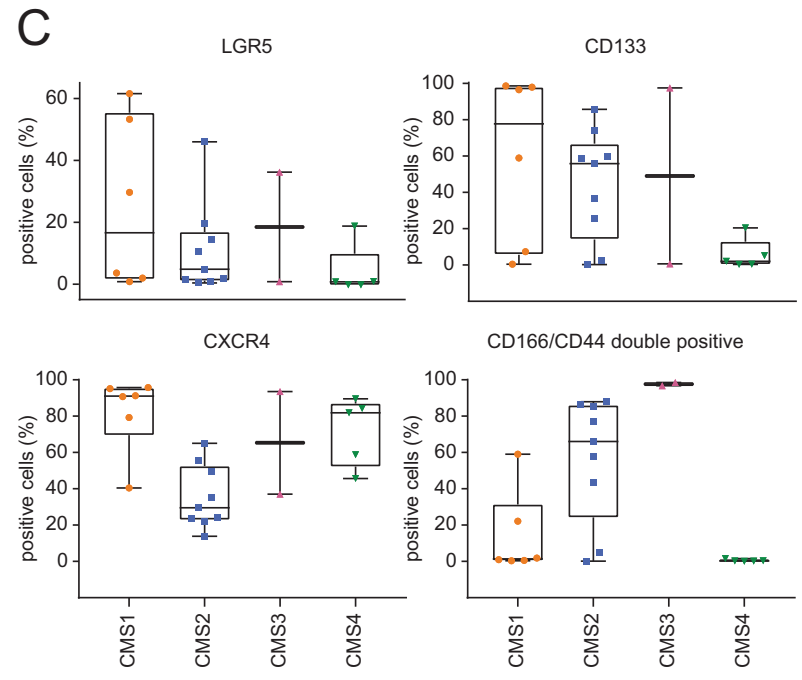

Fig. 5 Geneset enrichment analysis subtypes in CRC cell lines and primary cultures ( $n=29$ and $n=33$ respectively).a Geneset enrichment analysis shows CMS specific activation of pathways and processes in cell cultures, similar to those in patient tumours. b Gene

expression of CDX2, ZEB1, FRMD6 and HTR2B per subtype in CRC cell lines $(n=26)$ c FACS stainings for cancer stem cell marker LGR5, CD133, CXCR4 and CD166/CD44 double positive cells per subtype in CRC cell lines $(n=22)$ 
overrepresented at the expense of CMS3 and to a lesser extent CMS1 and CMS4. Moreover, a lower incidence of MSI+ cultures was observed compared to cell lines, suggesting that primary cultures do not display a bias for MSI + . To assess whether primary cultures faithfully represent the heterogeneity found in patient tumours, we compared expression profiles of eight available patient tumourprimary culture pairs (Supplementary Fig. S6). In all but one case primary cultures clearly recapitulate patientspecific expression patterns, similar to what has been reported previously [21]. We also compared CMS stratification of CRC cell lines and primary cultures with the CRIS tumour classification (Supplementary Fig. S7) [36]. As previously described, particular CMS subtypes preferentially map to certain CRIS subtypes, but significant variability is also detected between these two classification methods. Together our data indicate that CMS stratification of primary cultures and cell lines resulted in an overall set of 62 classified CRC cultures (Fig. 4b, Supplementary Table S1), which together provide a platform for further study of subtype-specific features and drug resistance (Supplementary Fig. S1B).

\section{Geneset enrichment analysis (GSEA)}

Several pathways and processes are differentially active between subtypes when analysing patient gene expression data [12]. These functional associations were largely recapitulated in established and primary CRC cultures (Fig. 5a). Although enrichment of typical infiltration dependent signatures, such as immune cell infiltration in CMS1, were not observed, metabolic dysregulation was evident from GSEA in CMS3 and epithelial enrichment observed in CMS2 and CMS3. Moreover, VEGFR and TGF- $\beta$ pathway activation as well as a clear mesenchymal expression pattern was observed in CMS4, likely linked to an EMT phenotype. Together this confirms that typical CMS features were to a large extent retained in CRC cultures. To corroborate these data we looked at CDX2, ZEB1, HTR2B and FRMD6, which were previously shown to stratify MSS+ epithelial from mesenchymal tumours [37]. In agreement with this notion, expression of these genes varied strongly between cell lines (Fig. 5b), but was largely in line with the subtypespecific expression observed in patients, confirming the presence of distinct CMSs in CRC cultures.

Next to a difference in expression of these 4 specific markers, WNT pathway activity as well as a distinction in CSC-derived signatures were observed between epithelial/ CMS2 and mesenchymal/CMS4 tumours [33,38] and this was maintained in cell cultures as indicated by GSEA
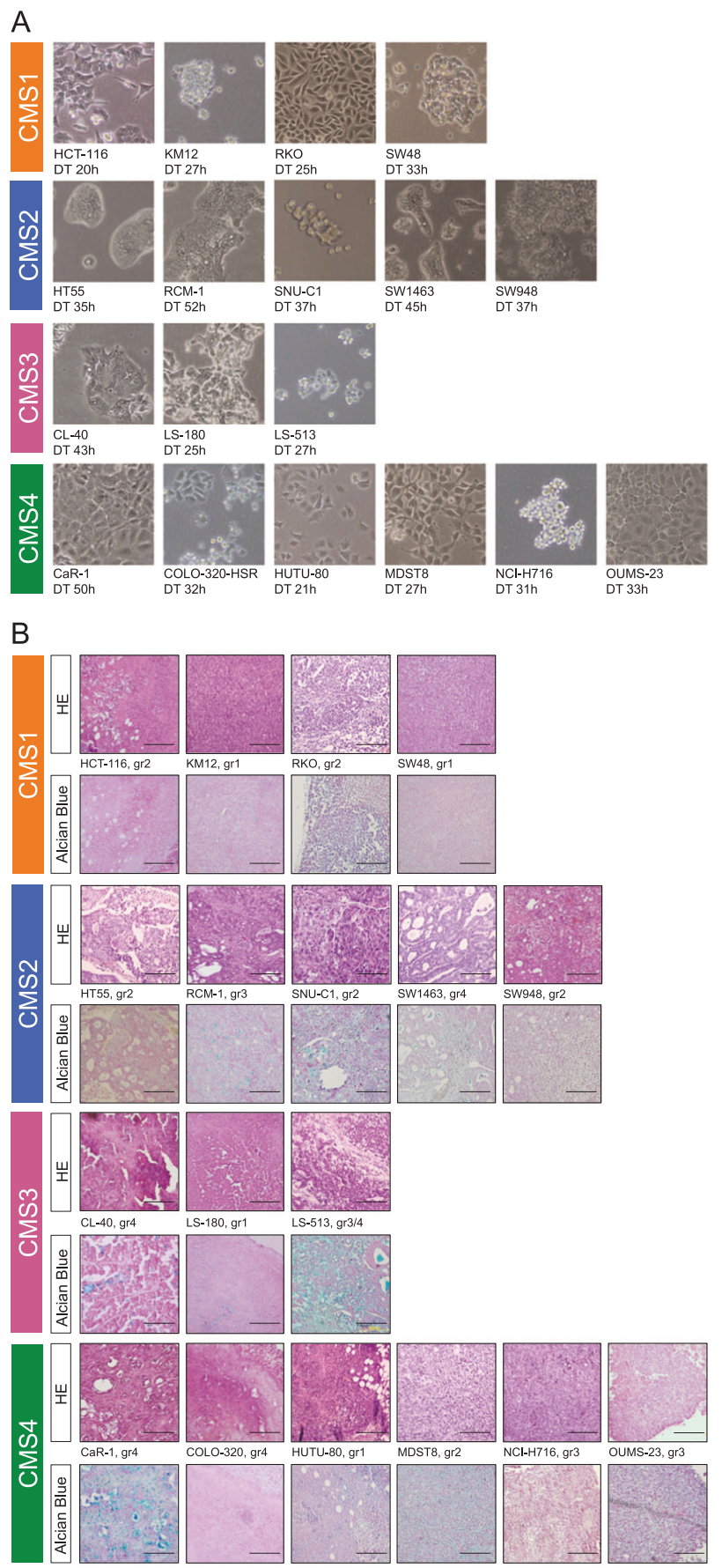

Fig. 6 Morphology and growth dynamics of selection of CRC cell lines $(n=18)$ in vitro and in vivo a Morphology and growth dynamics of $18 \mathrm{CRC}$ cell lines in culture representing distinct Consensus Molecular Subtype (CMS). Doubling Time (DT) reflects the average time needed to achieve one doubling over a course of three doublings. Picture were made with 20x magnification. b HE and Alcian blue staining of xenografts of the 18 selected cell lines. Time to reach a tumour size of $100 \mathrm{~mm}^{3}$ is stated after each cell line name in 4 groups (gr), namely group $1<20$ days, group 2 between 20-30 days, group 3 between 30-40 days and group $4>40$ days. Scale bar, $200 \mu \mathrm{m}$ 
(Fig. 5a). To analyse whether these differences were also evident at the protein level, FACS analysis on several commonly used CSC markers, such as CD133, LGR5 and CD166/CD44, was performed. Strikingly, a clear distinction between CMS2/3 and CMS1/4 cultures was evident when analysing CD166/CD44 expression, which was virtually undetectable in the CMS1/4 cultures (Fig. 5c). Similarly, LGR5 and CD133 marker expression was low to absent in CMS4, while expressed in the majority of cell lines belonging to the other subtypes. Vice versa, very high expression of CXCR4 was detected in CMS4 in comparison to CMS2, which has been associated with migration or migratory CSC, but may also regulate EMT [39], both features of CMS4. Importantly, this distinction was also detected in a set of primary cultures with no or low expression of CD133 and LGR5 in CMS4, but not CMS2 lines (Supplementary Fig. S8).

\section{Growth dynamics of cell lines and xenografts}

To gain further insight into in vitro differences between subtypes, the selected 18 cell lines, representing all $4 \mathrm{CMSs}$, were explored for their morphology and growth dynamics. Morphology of cell lines was distinct, also within subtypes (Fig. 6a). CMS2 and CMS3 cell lines, however, tended to grow in more dense colonies than CMS4 cell lines, which showed a more dispersed elongated phenotype, a good example being MDST8. Likewise, a significant variation between cell lines was observed regarding growth dynamics, (Fig. 6a) with fast-growing cell lines (e.g. HCT-116, HUTU-80 \& LS180) and slow-proliferating lines (e.g. CaR-1, RCM-1 \& SW1463), but this did not align with a specific subtype.

To determine in vivo growth rate and histology, cell lines were injected subcutaneously in mice and time to reach a tumour size of $100 \mathrm{~mm}^{3}$ was measured (Fig. 6b). Growth rates in general seemed slightly faster for CMS1 lines, but no difference was observed for the other subtypes. H\&E and Alcian Blue staining of tumours showed very distinct and to some extent subtype-specific characteristics (Fig. 6b). For instance, CMS1 xenografts were poorly differentiated, while CMS2/3 tumours in general showed clear crypt-like structures. Combined these data suggest that several of the subtype-specific features are not only maintained in vitro but also in vivo.

\section{Drug sensitivity}

Differences between subtypes in treatment response have been reported $[6,26,27]$. However, preclinical models to study subtypes were largely lacking. To explore drug sensitivity in a subtype-specific manner, cell lines were treated with oxaliplatin and 5-FU. Classical cell viability assays were employed revealing a broad IC50 range without a
Table 1 IC50 for oxaliplatin and 5-FU of CRC cell lines

\begin{tabular}{lllll}
\hline Cell line & $\begin{array}{l}\text { IC50 Oxali } \\
(\mu \mathrm{M})\end{array}$ & $\begin{array}{l}\text { AUC } \\
\text { Oxali }\end{array}$ & $\begin{array}{l}\text { IC50 5-FU } \\
(\mu \mathrm{M})\end{array}$ & $\begin{array}{l}\text { AUC } \\
5-\mathrm{FU}\end{array}$ \\
\hline HCT-116 & 0.75 & 21.82 & 1.89 & 14.27 \\
KM12 & 3.31 & 48.99 & 1.78 & 39.48 \\
RKO & 0.76 & 17.99 & 2.07 & 23.08 \\
SW48 & 0.45 & 12.25 & 3.46 & 32.46 \\
HT55 & 0.37 & 13.84 & 1.05 & 24.79 \\
RCM-1 & 0.12 & 20.98 & 0.35 & 33.95 \\
SNU-C1 & 0.81 & 48.69 & 2.84 & 46.44 \\
SW948 & na & 25.01 & 1.57 & 45.45 \\
CL-40 & 0.62 & 27.02 & 1.41 & 36.35 \\
LS-180 & 0.37 & 23.56 & 4.62 & 29.85 \\
LS-513 & 0.19 & 17.02 & 0.54 & 23.58 \\
CaR-1 & 9.66 & 58.03 & 3.06 & 45.28 \\
COLO-320- & 0.69 & 19.16 & 1.08 & 26.09 \\
HSR & & & & \\
HUTU-80 & 0.82 & 7.17 & 3.62 & 22.75 \\
MDST8 & 0.77 & 24.86 & 1.90 & 24.87 \\
NCI-H716 & 4.76 & 40.44 & 8.23 & 63.93 \\
OUMS-23 & 0.80 & 15.35 & 0.73 & 58.23 \\
\hline
\end{tabular}

Table showing the Inhibitory Concentration 50 (IC50) and Aria Under the Curve (AUC) for oxaliplatin (Oxali) and 5-fluorouracil (5-FU) of the selected 18 cell lines using a cell viability assay. Cells were treated with 11 or 18 (final) concentrations ranging from $100 \mu \mathrm{M}-100 \mathrm{nM}$ and a control or $100 \mu \mathrm{M}-800 \mathrm{pM}$ and a control. CMS1: HCT-116, KM12, RKO, SW48, CMS2: HT55, RCM-1, SNU-C1, SW948, CMS3: CL40, LS-180, LS-513, CMS4: CaR-1, COLO-320-HSR, HUTU-80, MDST8, NCI-H716, OUMS-23. IC50 was defined as concentration were $50 \%$ of the maximum effect was reached

clear subtype-specific response (Table 1). However, maximal achievable cytotoxicity was quite different between cell lines, with some lines displaying less than $50 \%$ signal reduction and others close to $100 \%$, suggesting that a difference in the type of response to drug existed ranging from cell cycle arrest to cell death. To study this directly, the Nicoletti assay was employed, which allows cell cycle and apoptotic cell death analysis simultaneously. Upon 5-FU treatment the majority of cell lines arrested in G1/S with a concomitant decrease in G2/M. In addition, a low but significant level of apoptosis was induced in several cell lines belonging to CMS1-3. However, no or very low levels of apoptosis were detected in CMS4 lines, suggesting that these resisted 5-FU-induced apoptosis. This difference was also evident when analysing the impact of oxaliplatin as in general CMS4 lines were more resistant to oxaliplatininduced apoptosis than CMS2 cell lines (Fig. 7a, Supplementary Fig. S9). Combined the observations indicated a selective resistance of CMS4 lines to chemotherapyinduced apoptosis. We attempted to further elucidate the molecular underpinnings of the differential apoptotic response by correlating this response to expression of $\mathrm{BCL}$ - 
2 family members, which play a key role in regulating apoptosis. However, no single BCL-2 family member showed a significant association with apoptotic response
(Supplementary Fig. S10a). To move beyond the level of single genes we also analysed sets of related genes (GSEA) and found that, among others, both Wnt and Notch
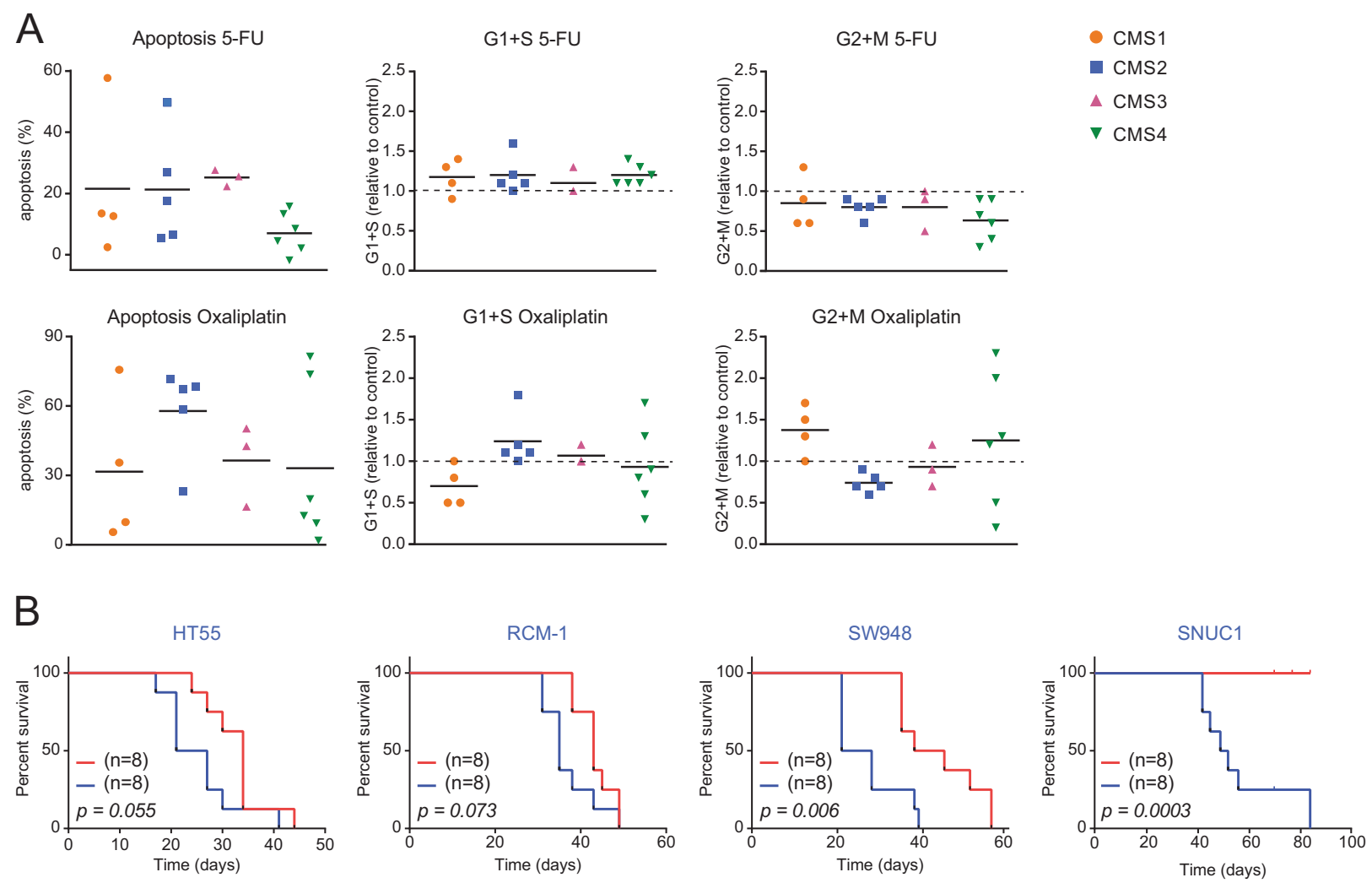

CMS2 combined
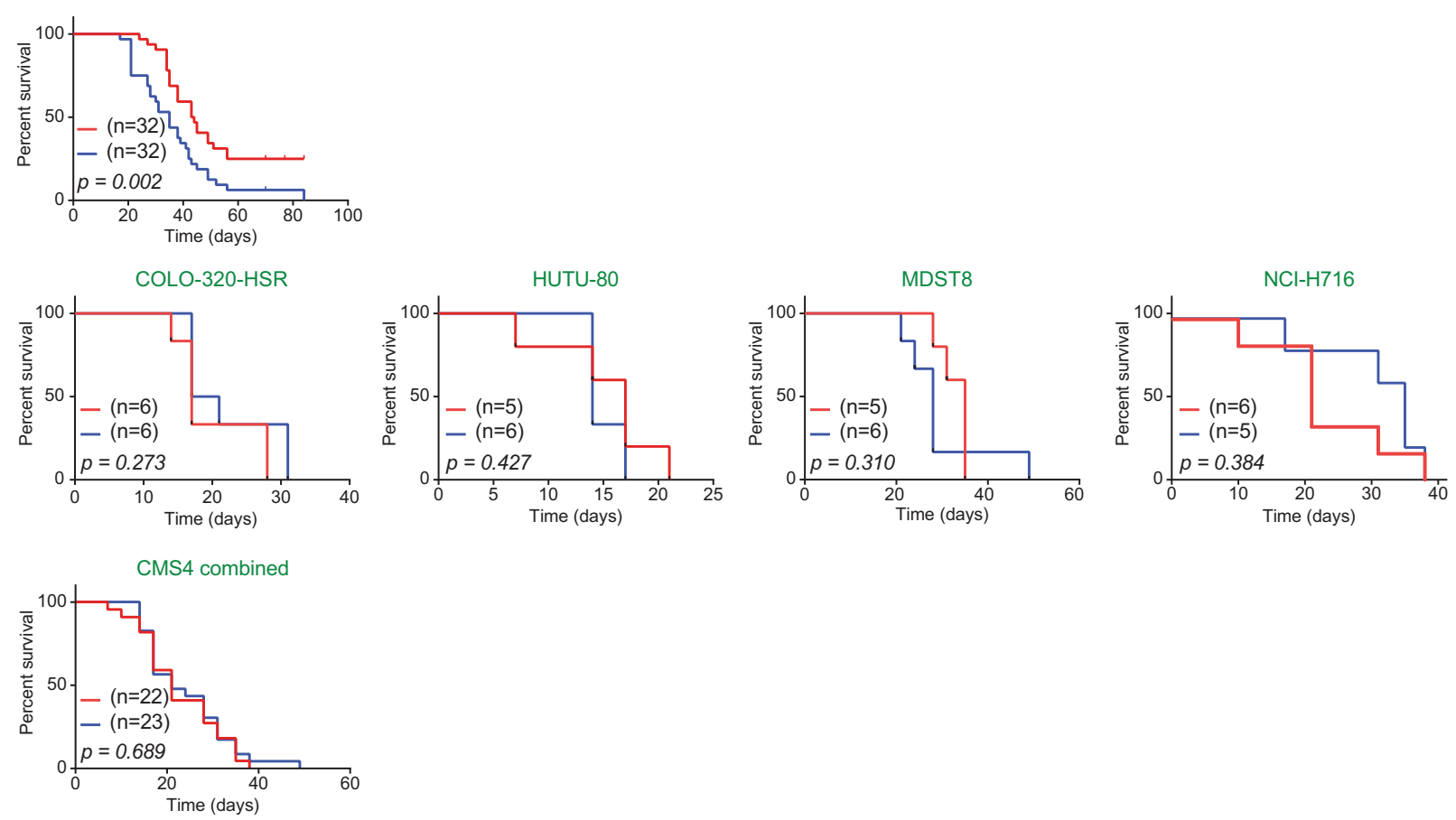

- Oxaliplatin + 5-FU

- PBS 
Fig. 7 Drug sensitivity in vitro and in vivo of CRC cell lines a Drug sensitivity assessed by Nicoletti assay and measured by apoptosis and cell cycle arrest for 18 CRC cell lines. Dots represented values of individual cell lines classified in that CMS. Apoptosis is the percentage of cells in apoptosis after treatment with oxaliplatin or 5-FU distracted by the percentage of apoptosis in control samples. G1/S is calculated by the fraction of cells in G1/S from total cells in cycle (sum of G1, S, $\mathrm{G} 2, \mathrm{M})$ as a ratio to cells in $\mathrm{G} 1 / \mathrm{S}$ from total cells in cycle in untreated control sample $\mathrm{G} 2 / \mathrm{M}$ is calculated by the fraction of cells in $\mathrm{G} 2 / \mathrm{M}$ from total cells in cycle $(\mathrm{G} 1+\mathrm{S}+\mathrm{G} 2+\mathrm{M})$ as a ratio to cells in $\mathrm{G} 2 / \mathrm{M}$ from total cells in cycle control sample. b Drug sensitivity in vivo measured by survival assay for $8 \mathrm{CRC}$ cell lines that were injected subcutaneously. Four CMS2 (HT55, RCM-1, SNU-C1 and SW948) and four CMS4 (COLO-320-HSR, HUTU-80, MDST8 and NCIH716) classified cell lines were in vivo treated with a combination of oxaliplatin and 5-FU (red line) or PBS (blue line) as control. Drug sensitivity was measured by survival differences between treated and control groups and analysed with Log-rank tests. The two graphs on the right represent the combined subgroup effect. Abbreviations: $C M S$ Consensus Molecular Subtype, PBS Phosphate-buffered saline, 5FU5-Fluorouracil

pathways were up-regulated in oxaliplatin as well as 5-FU sensitive cell lines, whereas the P53 pathway was downregulated in 5-FU sensitive cell lines (Supplementary Fig. S10b). Interestingly, when looking at gene mutations we found that Wnt pathway mutations were also associated with oxaliplatin sensitivity (Supplementary Fig. S10c) strengthening the suggestion that Wnt pathway activity is associated with drug sensitivity.

To determine whether in vitro differences were relevant for in vivo response to chemotherapy, four CMS2 and four CMS4 lines were injected subcutaneously into immunedeficient mice. Combination treatment with 5-FU/oxaliplatin was performed when tumours reached a size of 100 $\mathrm{mm}^{3}$. Overall, chemotherapy had limited impact on tumour growth and survival of mice in the majority of cell lines tested, in line with the relatively high concentrations needed to kill cells in vitro. Nevertheless, a clear delay in tumour growth was observed for CMS2, but not CMS4 cell linederived tumours, which were not affected by chemotherapy at all (Fig. 7b). These data therefore support the idea that CMS4 tumours are chemotherapy-resistant. More importantly, the current cell line repository indicates that CMS can be detected in vitro and in PDX models and allow for subtype-specific analysis of drug responses.

\section{Discussion}

Using a plethora of CRC models, we establish that the heterogeneity observed in patients extends to the experimental models with all observed variation in methylation events (CIMP, miR200, Wnt targets) and mutations in key signalling pathways. As reported before [31,40], there is bias towards MSI+ in the cell lines and a lowered incidence of PI3K/TGF $\beta$ pathway mutations, which is not detected in primary cultures or PDX models, which may relate to the difference in culture conditions.

Nevertheless, the most important aspect of our findings is the identification of all CMSs in CRC cell lines, primary cultures and PDX models. Previous analyses failed to identify the CMS4 subtype in PDX, which was explained by the fact that mesenchymal features were not derived from tumour cells, but from stroma and were therefore not recapitulated in PDX models or cell cultures [24,30]. However, other studies indicated mesenchymal marker expression, such as ZEB1, in tumour epithelial cells of CRC patients [37]. Secondly, several CRC cell lines have been shown to express mesenchymal markers, which can be regulated by miR200 methylation [34], suggesting that mesenchymal features are not unique to stroma. In agreement, 29 of 43 cell lines could be CMS stratified with high confidence, including CMS4. Combining all cultures, we observe that each CMS is detected, but that CMS3 is clearly underrepresented in favour of CMS2 as compared to patient cohorts. The underlying reason for this decrease is not clear, but could relate to a more stringent metabolic wiring that may not be compatible with culture. As expected, CMS1 is enriched for MSI samples supporting the robustness of our classification. Nevertheless, one could argue that stratification of any dataset is possible based on median expression values and that CMS cell line assignment is not related to the original tumour. However, this criticism was circumvented by employing a biobank to link the CMS of patients with PDXs. This approach validated that subtypes are (1) demarcated by tumour-intrinsic differences and can also be detected in PDX models and are (2) maintained upon passaging into PDX models and in cultures. Using an epithelium specific signature, CMS4 tumours are identified, not only in patients, but also in PDX models and above all results in an almost identical classification of established cell lines. This validates subtype-specific epithelial features, which are maintained in cell lines and PDX models as well as reported biological differences.

Recently, five tumour-intrinsic subtypes in CRC cell lines were reported called CRIS-(A-E) [36]. Significant overlap exists between CMS and CRIS, but also clear differences and both classifications will be vital to study tumour subtype-specific treatments. Although established cell lines have been in culture for a long time and may have lost patient characteristics, our data argue that gene expression profiles are rather stable and can even be used to validate the identity of cell lines. We therefore employed these CMS stratified cell models to analyse the cytotoxic effect of chemotherapy. As previously reported [41], 5-FU caused a G1/ $\mathrm{S}$ arrest independent of subtype. In addition, apoptosis was induced by 5-FU, specifically in CMS1-3 cell lines, whereas apoptosis was low to absent in CMS4 lines. Similarly, after 
treatment with oxaliplatin a clear distinction in apoptosis resistance was evident between CMS4 and CMS2 cell lines. The poor response to chemotherapy in CMS4 cell lines is in line with the lack of response to oxaliplatin in CMS4 patients [26]. Vice versa, the specific sensitivity to oxaliplatin by a CMS2 subset, which was reported in that study, appears to be mirrored by the cell line responses. This difference was also observed in vivo with CMS2 cell lines showing a greater sensitivity compared to CMS4.

To conclude, the overall goal of this study was to determine whether CRC cell lines, primary cultures and PDX models recapitulate the heterogeneity observed in patient tumours. Our data provide an overarching insight into this heterogeneity and provide strong support for the presence of subtypes in CRC models. These differences should be considered carefully when analysing drug efficacy screens, pathway activity and biological phenotypes in CRC. This study therefore provides a unique platform for $\mathrm{CRC}$ researchers and serves as a starting point to identify CMS-specific features that will hopefully pave the way to subtype-specific therapies.

\section{Materials and methods}

\section{Colorectal cancer cell lines}

Eighteen CRC cell lines were included for in vitro and in vivo experiments. All were kindly provided by Sanger Institute (Cambridge, UK; authenticated by STR Genotyping). Cell lines RKO, SW48, HT55, SW948, SW1463, CL-40, LS-180, CaR-1, HUTU-80 and OUMS-23 were cultured in Dulbecco's modified Eagle's medium/F-12 medium with L-glutamine, 15 mM HEPES (Thermo-Fisher Scientific, Bleiswijk, The Netherlands) with added foetal bovine serum (Lonza, Breda, The Netherlands) and penicillin and streptomycin. HCT-116, KM12, RCM-1, SNUC1, LS-513, COLO-320-HSR, MDST8 and NCI-H716 were cultured in RPMI 1640 with L-glutamine, $25 \mathrm{mM}$ HEPES (Thermo-Fisher Scientific, Bleiswijk, The Netherlands) added fetal bovine serum, penicillin and streptomycin, 1\% D-glucose solution plus (Sigma-Aldrich, Zwijndrecht, The Netherlands) and $100 \mu \mathrm{M}$ sodium pyruvate (Life Technologies, Bleiswijk, The Netherlands). All cultures were maintained in humidified $37^{\circ} \mathrm{C}$ $5 \% \mathrm{CO}_{2}$ incubators and cells were regularly tested for mycoplasma infection. Microscopic bright-field images were taken on a Leica DMi1 microscope. After growth RNA was isolated from the cell lines for gene expression analysis.

\section{Short tandem repeat (STR)-profiling}

A total of $10 \mathrm{ng}$ of gDNA per cell line was amplified in a PCR reaction using Powerplex® 16 system (Promega, Leiden, The Netherlands) according to manufacturer's instructions. Thermal cycling was performed as follows: $95^{\circ} \mathrm{C}$ for $15 \mathrm{~min}, 96^{\circ} \mathrm{C}$ for $1 \mathrm{~min}, 10$ cycles of $94^{\circ} \mathrm{C}$ for 30 $\mathrm{s}, 60^{\circ} \mathrm{C}$ for $30 \mathrm{~s}, 70^{\circ} \mathrm{C}$ for $45 \mathrm{~s}, 20$ cycles of $90^{\circ} \mathrm{C}$ for $30 \mathrm{~s}$, $60{ }^{\circ} \mathrm{C}$ for $30 \mathrm{~s}$ and $70{ }^{\circ} \mathrm{C}$ for $45 \mathrm{~s}$, and $60{ }^{\circ} \mathrm{C}$ for $30 \mathrm{~min}$. Next, PCR product was mixed with Internal Standard Lane 600 (ILS600) and Hidi Formamide. Results were analysed using Genescanning ${ }^{\circledR}$ software (Applied Biosystems). The following loci were amplified: Penta E, D18S51, D21S11, TH01, D3S1358, FGA, TPOX, D8S1179, vWA, Amelogenin, Penta D, CSF1P0, D16S539, D7S820, D13S317 and D5S818.

\section{Doubling time determination}

Cells were seeded in T25 flasks in $4 \mathrm{ml}$ of medium at a density that would allow for at least three doublings to occur. At each indicated time point, both medium and washing PBS were collected from one T25 flask and cells were harvested using trypsin. PBS was used to rinse the flask after trypsinised cells were aspirated to ensure all cells present in the flask were collected. Cells were counted using trypan blue and total number of live cells per flask was calculated. Medium in flasks remaining in the experiment was refreshed every 3 days. Doubling times listed reflect the average number of hours needed to achieve one doubling over a course of three doublings.

\section{Primary cell cultures}

Spheroid cultures were isolated from different patients with colorectal cancer in accordance with the rules of the medical ethical committee of the AMC, University of Palermo and UMC Utrecht. CO108, CO115, CO123, CO133, CO147, CO152 and LM23 were established in the AMC, Amsterdam. AG2, CCO9, CO01, DA13, LR13, LR14, RC511 and ME59 originated from Palermo, Italy. All these primary cultures, jointly called the biobank were cultured under ultralow-adherent condition (Corning, Amsterdam, The Netherlands) as described previously [42] in cancer stem cell medium. In summary, advanced DMEM/F12 was used supplemented with D-glucose $0.15 \%$ (Sigma), HEPES $5 \mathrm{mM}$ (Life Technologies), penicillin/streptomycin (PS), Heparin $2 \mu \mathrm{g} / \mathrm{ml}$ (Sigma), Insulin $10 \mu \mathrm{g} / \mathrm{ml}$ (Sigma), $\beta$-mercaptoethanol $100 \mu \mathrm{M}$ (Sigma), and trace element B and C (Fisher Scientific) with freshly added human EGF 20ng/ml (Pepropech) and FGF 10ng/ml. For the primary culture panel of the Hubrecht Institute (P6T, P7T, P8T, P9T, P11T, P14T, P16T, P17T, P18T, P19Tb, P20T, P23T, P24Ta, P24Tb, P25T, P26T, P28T and P31T), cells were cultured as organoids in Matrigel matrix (BD Biosciences, Breda, The Netherlands) and cultured in the medium as previously described. In short, cancer stem cell medium was used as described above except for SB202190 10 $\mathrm{MM}$ (Sigma) and B27 1\% (Invitrogen) which were added freshly and no FGF 
was added. After growth RNA was isolated from the primary cultures and gene expression analysed.

\section{PDX establishment}

Tumour material from patients who underwent surgery at the Academic Medical Center (Amsterdam, the Netherlands) or Flevo hospital (Almere, the Netherlands) was obtained within $3 \mathrm{~h}$ after surgery. Prior to transplantation, tumour fragments were washed several times in Hank's Balanced Salt Solution (HBSS; GIBCO, Grand Island, NY, USA) containing $100 \mathrm{U} / \mathrm{ml}$ penicillin and $100 \mu \mathrm{g} / \mathrm{ml}$ streptomycin (GIBCO, Grand Island, NY, USA), $10 \mathrm{mg} / \mathrm{ml}$ gentamycin (Lonza) and 2,5 $\mu \mathrm{g} / \mathrm{ml}$ amphotericin-B (Lonza, Basel, Switzerland). Then, subcutaneous implantation was performed in the flank of 5 to 6 week-old Hsd: Athymic-Nude Foxn1 mice (Envigo, Horst, the Netherlands) or NOD/SCID IL2R gamma ${ }^{-l-}$ (NSG)(own breeding) mice weighing from $18-25 \mathrm{~g}$ that were anesthetised by a mixture of isoflurane/ oxygen. Successful engraftment of the tumour and subsequent growth were monitored by weekly measurement of the implantation sites. The serial engraftments of each given tumour were conducted on 2 nude or NSG mice when the tumours reached a volume of $200-1000 \mathrm{~mm}^{3}$. After growth RNA and DNA were isolated from the tumours (patient and PDXs) and gene expression was analysed.

\section{Gene expression profiling}

Microarrays expression profiles were obtained using the GeneTitan $^{\text {tm }}$ MC system from Affymetrix according to the standard protocols of the Cologne Center for Genomics (CCG), University of Cologne, Germany as previously described [43]. The datasets have been deposited in the Gene Expression Omnibus (GEO) repository under the accession numbers GSE100478 (18 CRC cell lines), GSE100549 (15 primary cell cultures from the AMC/Palermo biobank) GSE100479 (18 primary cell cultures from the Hubrecht Institute) and GSE100480 (21 patients and 34 PDXs). Each dataset was normalised using the Robust Multi-array Average (rma) method as implemented in the affy $\mathrm{R}$ package (version 1.52.0). For GSE100549 and GSE100480 we used the ComBat algorithm as implemented in the sva $\mathrm{R}$ package (version 3.22.0) to remove batch effects. Probes were annotated with the hgu133plus2.db R data package (version 3.2.3).

\section{Cell line stratification}

A panel of CRC cell lines $(n=43)$ was CMS and CCS stratified with 5 different gene expression classifiers using expression data from 5 different datasets (Supplementary Fig. S3). Three classifiers were developed by the ColoRectal Cancer Subtyping Consortium (CRCSC) [12] and the CCS classifier as well as the actual classification of the cell lines were described in Melo et al. [6]. Another classifier was newly developed specifically for cell line classification (CLC) which is described in the next paragraph.

The combination of 5 classifiers and 5 datasets resulted in a maximum of 11 classifications per cell line (Fig. S3). Not every cell line was present in every dataset, therefore the number of classifications per cell line varied. These results were consolidated into a single classification where at least $66 \%$ of classifications had to concur to arrive at a CMS assignment. Ultimately 29 of 43 cell lines received a CMS label. For the colorectal cancer subtype (CCS) classification we considered a CCS1 label to be in accordance with CMS2 or CMS3, CCS2 with CMS1 and CCS3 with CMS4.

\section{CRCSC classifiers}

Raw microarray data were downloaded from five independent cohorts, including the Cancer Cell Line Encyclopaedia [17] (CCLE, GSE36133), Wagner et al. [44] (GSE8332), Arango et al. [45] (Mariadason, GSE35566), Garnett et al. [16] (Sanger, GSE68950) and Medico et al. [31] (Medico, GSE59857). For the CCLE, Wagner and Mariadason sets, we first normalised and summarised gene expression profiles using frozen robust multiarray analysis (fRMA) [46]. Non-biological batch effects were detected between these three data sets using principal component analysis, and corrected using ComBat [47]. For the Medico set, non-normalised data were generated by the Illumina GenomeStudio software version V2011.1 as described in Medico et al. [31], and were further Loess normalised using the Lumi R package [48]. For the Sanger set, we downloaded the raw microarray data from ArrayExpress, and performed normalisation using Robust Multi-array Average (RMA) method. The previously established CCS classification on the normalised CCLE dataset [33] was subsequently subjected to the CMS classifier established by the ColoRectal Cancer Subtyping Consortium (CRCSC) [12]. The pre-processed gene expression profiles for the Sanger set, Medico set and the combined set (CCLE, Wanger and Mariadason) were standardised per gene also subjected to the CMS classifier established by the CRCSC for classification respectively. Finally, the network classification was performed on the CRC cell line set. In short all individual classifications of the CRCSC were applied to the CRC cell line set and a network was constructed in a similar fashion as for the patient stratification. CMS classes were subsequently allocated to the cell lines based on the network clustering.

\section{Cell line classifier (CLC)}

Raw microarray data were downloaded for three CRC cell lines datasets (GSE36133, GSE68950 and GSE59857) as well as a CRC tumour cohort [7] (GSE39582). GSE36133, GSE68950 and GSE39582 were normalised and gene 
expression summarised using robust multiarray analysis (rma) [49], GSE59857 was normalised as described in the previous paragraph. For GSE39582 batch effects were corrected using ComBat. Given the inherent differences between bulk tumours and cell line cultures we included a "correlation of correlations" step, described in Guinney et al. [12], to select features (probesets) exhibiting similar patterns of co-expression in tumours and cell lines. This was implemented by calculating the Pearson correlation of each probeset with every other probeset in a CRC tumour dataset (GSE39582) and a dataset containing CRC cell lines (GSE36133). This resulted in two vectors of correlation coefficients for every probeset, one for the tumour dataset and one for the cell line dataset. As a final step the Pearson correlation coefficient of these two vectors is calculated to get a single correlation of correlations score for each probeset. Only probes with a correlation of correlations score $>0.25$ were used for the subsequent steps $(n=15,327)$.

final classifier construction $(n=133)$.

The classifier is a support vector machine (SVM) that was constructed using the standardised expression of the 133 probesets in the GSE39582 dataset. We employed the SVM implementation of the e1071 R package [51] using a linear kernel. Only samples with known CMS status were used for classifier construction. GSE36133 was profiled on the same Affymetrix U133 Plus 2.0 platform as GSE39582 and was classified using the expression of the same probesets used in the classifier construction. GSE68950 however, was profiled on the older Affymetrix U133A platform that contains only a subset of the probesets present on U133 Plus 2.0 platform. Missing probesets were replaced by calculating the Pearson correlation coefficient of the missing probeset with other probesets present on the U133A platform (using GSE39582) and selecting the probeset with the highest coefficient as a replacement for classifier construction. In case of GSE59857, which was profiled using a non-Affymetrix array platform (Illumina HumanHT-12 V4.0 expression beadchip), we used gene mapping for cross-platform classification (all genes in the classifier were present on both platforms). Expression values were standardised within each dataset before classification.

\section{Gene/pathway mutations}

Lists of cancer driver genes were acquired from the COSMIC [52] (cancer_gene_census.csv, release v80) and IntOGen [53] (intogen_cancer_drivers-2014.12.zip) databases. Cell line mutational data were downloaded from COSMIC for 42 cell lines (CosmicCLP_MutantExport.tsv, release v80) and from CCLE for 1 cell line (OUMS-23) absent from the COSMIC database (CCLE_hybrid capture1650_hg19_NoCommonSNPs_NoNeutralVariants_CDS_ 2012.05.07.maf). Mutations predicted to be neutral by the
FATHMM algorithm [54] were filtered from the COSMIC database. To determine whether the cell line mutations were driver or passenger mutation a pan-cancer database of nonsilent mutations was compiled (i.e. PCD) using the IntOGen (intogen_driver_mutations_catalog-2016.5.zip) and Cosmic (CosmicCompleteTargetedScreensMutantExport.tsv, release v80) databases. All mutations predicted to be neutral by the FATHMM algorithm were filtered from the COSMIC database. Activating mutations in cell lines were deemed driver events only when present in the PCD and designated a 'known driver' by the IntOGen or alternatively when detected in more than 50 patient tumours. Non-activating mutations were retained as driver events when a mutation in the same exon was present in the PCD.

\section{PDX classifier}

In addition to a cell line classifier (CLC) we also developed a classifier specifically aimed at stratifying patient-derived xenografts (PDX). This PDX classifier is a step-wise process of three separate classifiers which combine, as shown in Fig. 3, to CMS stratify each PDX model. Two of the classifiers (the CMS1 and CMS3 classifiers) were constructed similar to the CLC classifier with the following exceptions. The correlation of correlations probeset selection step was performed using the GSE100480 set of matched patients and PDX models. The correlation of correlations was calculated using the patients on the one hand and the PDX models on the other hand, resulting in the selection of 15,459 probesets. Subsequently a CMS1 SVM classifier was constructed using GSE39582 designed to stratify CMS1 from non-CMS1 and similarly a CMS3 classifier that can discriminate between CMS2 and CMS3 (since CMS1 and CMS4 PDX models are stratified before application of the CMS3 classifier). The third classifier was constructed differently due to the fact that CMS4-specific expression is dominated by the increased accumulation of tumour stroma [29,30], which is replaced by murine tissue in PDX models [35]. The stromal expression component in PDX models is therefore not detected, or only partially detected, by the Affymetrix microarray platform, which is designed against the human transcriptome. To circumvent this limitation we employed two related FACS sorted datasets (GSE39395 and GSE39396) that report on expression profiles of epithelial tumour cells and stromal fibroblasts from 14 patients [55]. Using these datasets we identified differential gene expression between the tumour and stromal compartment using the limma R package. Subsequently, we set a number of thresholds (log fold change $>2$, adjusted $P<0.001$ and expression $>7$ in the tumour compartment of 1 or more patients) to define a set of genes $(n=389)$ that were selectively expressed in the tumour epithelial compartment and at least 4-fold lower 
in the stromal compartment (Supplementary Fig. S11a). To ensure that these genes were also expressed in cell lines we checked the expression in a CRC cell line dataset (GSE36133) and only retained genes with an expression $>8$ in at least one cell line resulting in a set of 359 tumour specific genes (Supplementary Fig. S11a). The expression profile of a patient's tumour is a combination of expression coming from the tumour and the stromal compartments. Tumours with a high stromal content (e.g. CMS4, Supplementary Fig. S11b) will therefore have lower expression levels of tumourspecific genes ('stromal dilution' effect), an effect entirely absent in the PDX models. To ensure independence of the effects of stromal dilution, we normalised the expression of the 359 tumour-specific genes to the within sample mean of those genes (Supplementary Fig. S11c). After normalisation, these tumourspecific genes were used to construct a classifier designed to distinguish CMS4 from CMS2/3 using GSE39582. The patients from which the PDX models were derived were stratified using a classifier trained on GSE39582, similar to the PDX classifier but without the preceding feature selection steps.

\section{Cell line trained classifier}

After establishing classifications for the panel of 29 CRC cell lines, we used this panel as a gold standard to construct a cell line trained CMS classifier. We combined the expression profiles present in GSE36133 $(n=$ $25)$ and GSE100478 $(n=1)$, using ComBat to remove batch effects and retained one probeset per gene (highest mean expression). We next selected probesets with a standard deviation $>0.6$ and a $\log 2$ expression $>11$ in at least one cell line. The most relevant probesets were selected using cross-validation (1000 iterations) as described before and a 65-gene SVM classifier was constructed using standardised expression (Supplementary Table S2). This classifier was then applied to the primary cell cultures of GSE100479 (Hubrecht Institute) and GSE100549 (AMC/Palermo biobank).

\section{GSEA and CRIS classification}

GSEA [56] and CRIS classification were performed on dataset containing cell line and primary culture expression profiles from GSE100478, GSE36133, GSE68950, GSE100479 and GSE100549 (Supplementary Table S3). Batch effects were removed using ComBat. The GSEA was performed with the use of the GSA R package [57] using 10,000 permutations and the genesets mentioned in Supplementary Table S4 and S5. The CRIS classification was performed using the $\mathrm{R}$ package provided by Isella et al.
(CRIS classifier) and CRIS labels were assigned to cell cultures with a Benjamini-Hochberg FDR $\leq 0.2$, as recommended by Isella et al.

\section{Cell viability assays}

To analyse cell viability, cells were seeded in a 96-well plate in triplicate in $75 \mu \mathrm{l}$ of medium. After $24 \mathrm{~h}, 25 \mu \mathrm{l}$ of drug-containing media was added to the cells (oxaliplatin (Sigma-Aldrich, Zwijndrecht, The Netherlands), 5fluorouracil (5-FU) (Sigma-Aldrich, Zwijndrecht, The Netherlands) both dissolved in DMSO) in 11 or 18 different (final) concentrations ranging $100 \mu \mathrm{M}-100 \mathrm{nM}$ or 100 $\mu \mathrm{M}-800 \mathrm{pM}$ in a 1:2 dilution and a control. After $72 \mathrm{~h}$ of incubation with the agents, $20 \mu$ of Cell Titer Blue (Promega, Leiden, The Netherlands) was added and incubated for $3 \mathrm{~h}$ in $37^{\circ} \mathrm{C} 5 \% \mathrm{CO}_{2}$ incubators protected from light. Fluorescence signal was measured by fluorescence reader (Biotek). Background fluorescence was subtracted from each data point, and cell viability at each concentration was expressed as a fraction of untreated control wells. For this experiment, at least three independent replicates were performed. IC50 and Area Under the Curve (AUC) calculations were done using GraphPad Prism version 7. IC50 was defined as the concentration where $50 \%$ of the maximum effect was reached.

\section{Cell cycle and apoptosis analyses}

Cells were seeded in a 6-well plate with 30,000 or 100,000 per well, depending on their doubling time. After $24 \mathrm{~h}$ medium was refreshed and oxaliplatin and 5-FU were added at a final concentration of $25 \mu \mathrm{M}$ and incubated for $72 \mathrm{~h}$. Cells, medium and washing-PBS were collected and cells were resuspended in Nicoletti buffer $(0.1 \% \mathrm{w} / \mathrm{v}$ Sodium citrate, $0.1 \% \mathrm{v} / \mathrm{v}$ Triton X-100 in demineralised water, $\mathrm{pH}$ 7.4, supplemented with $50 \mu \mathrm{g} / \mathrm{mL}$ propidium iodide before use). After overnight incubation at $4{ }^{\circ} \mathrm{C}$, samples were analysed using flow cytometry (FACS Canto II). The experiment was repeated at least three independent times. Calculations for cell cycle fractions were done as follows: $((\mathrm{G} 1+\mathrm{S}) /(\mathrm{G} 1+\mathrm{S}+\mathrm{G} 2+\mathrm{M}))_{\text {treated }} /((\mathrm{G} 1+\mathrm{S}) /(\mathrm{G} 1+\mathrm{S}+$ $\mathrm{G} 2+\mathrm{M}))_{\text {control }}$ and $((\mathrm{G} 2+\mathrm{M}) /(\mathrm{G} 1+\mathrm{S}+\mathrm{G} 2+\mathrm{M}))_{\text {treated }} /$ $((\mathrm{G} 2+\mathrm{M}) /(\mathrm{G} 1+\mathrm{S}+\mathrm{G} 2+\mathrm{M}))_{\text {control. }}$.

Correlation analysis and GSEA were performed using the GSE100478 dataset containing 18 CRC cell lines. GSEA was done using the GSA R package [57] with 1000 permutations and the hallmark genesets from MSigDB [56].

\section{Mice experiments}

Animal experiments were approved by the animal welfare committee of the Academic Medical Center as required by 
Dutch law in LEX103093-1, LEX144AA1 and LEX140AB. Animals were housed in individually ventilated cages at the Animal Research Institute Amsterdam (ARIA-IWO).

For growth dynamic experiments, $1 \times 10^{6}$ human CRC cells were, after extensive washing with $\mathrm{PBS}+0.5 \%$ Bovine Serum Albumine (Sigma-Aldrich, Zwijndrecht, The Netherlands), injected subcutaneously in the right flank of the Hsd: Athymic-Nude Foxn1 mice (Envigo, Horst, The Netherlands) or NOD/SCID IL2R gamma-/- (NSG) (own breeding) mice. When tumours had reached a volume of $300 \mathrm{~mm}^{3}$, mice were sacrificed and xenografts were stored freshly frozen in $-80^{\circ} \mathrm{C}$ and in FFPE. For oxaliplatin/5-FU experiments, $1 \times 10^{6}$ human CRC cells were injected subcutaneously in the right flank of the NSG mice. When tumours had reached a volume of $50-200 \mathrm{~mm}^{3}$, treatment was started with intraperitoneal injection with oxaliplatin $0.25 \mathrm{mg} / \mathrm{kg}$ on days $3,10,18$ and 25 and $5-\mathrm{FU} 16 \mathrm{mg} / \mathrm{kg}$ on days $1-5$ and $15-20$ or as a control with PBS with a total time of treatment of four weeks. When tumours reached a volume over $1000 \mathrm{~mm}^{3}$ mice were sacrificed. Each treatment group consisted of 6 or 8 mice. Survival curves were generated using GraphPad Prism version 7 and analysed with Log-rank tests. $P$ values $<0.05$ were considered as significant.

\section{FACS stainings}

Cells were dissociated and for each staining 50,000 cells were pelleted and washed with PBS + BSA $1 \%$ for blocking unspecific antibody binding. Antibodies were diluted in PBS + BSA $1 \%$ in a total volume of $60 \mu$ and were added to the cells. Cells were incubated for $30 \mathrm{~min}$ at $4{ }^{\circ} \mathrm{C}$ and subsequently washed twice before FACS analysis (FACS Canto II). Cells incubated with unconjugated antibody were prepared for an extra incubation with labelled secondary antibodies again for $30 \mathrm{~min}$ at room temperature (cell lines) or $4{ }^{\circ} \mathrm{C}$ (primary cultures). Dead cells were excluded using propidium iodide or 7-AAD (Sigma-Aldrich, Zwijndrecht, The Netherlands). Isotype controls were used to determine non-specific background and channel compensation was performed on single-colour-stained samples. The following antibodies were used: CD133/1 PE (Miltenyi, AC133 / 130080-801, 1:100), CD166-PE (R\&D, MAB172, 1:100), CXCR4 (R\&D, MAB172, 1:300), LGR5 Biotine (BD, 562913 / 4D11F8, 1:100), Streptavidin APC-conjugated (eBioscience, 17431782, 1:500), $\alpha$-mouse-APC (BD, 550826, 1:500). Analysis was performed on FlowJo software.

\section{Immunohistochemical stainings}

Paraffin-embedded sections of $4 \mu \mathrm{m}$ were made from subcutaneously grown xenografted tumours. They were deparaffinised and rehydrated. Sections were stained for Haematoxylin/Eosin (HE) and alcian blue/nuclear red staining.

\section{CIMP analysis}

Genomic DNA (gDNA) from cell lines was isolated using the High Pure PCR Template Preparation kit (Roche, Basel, Switzerland), and bisulfite converted with the EpiTect Bisulfite Kit (Qiagen, Hilden, Germany) or EZ-DNA methylation Gold-Kit (Zymo, Irvine, CA, USA) according to the manufacturer's instructions. Genomic DNA from primary cultures was isolated using the Nucleospin Tissue kit (Bioke, Leiden, the Netherlands). TaqMan-based methylation-specific real time PCR (Methylight) was used to measure the CIMP status of all the cell cultures. Eight CIMP-specific markers (CACNA1G, CDKN2A, CRABP1, IGF2, MLH1, NEUROG1, RUNX3, SOCS1) and a control (ALU) were used in the assay. Primer and probe sequences have been described previously [58]. The ALU probe was labelled with a minor-groove binder non-fluorescent quencher (MGBNFQ; Applied Biosystems, Foster City, California, USA); other probes were labelled with a blackhole quencher (BHQ-1; Biosearch Technologies, Novato, California, USA). We performed the Methylight reaction on a Light Cycler 480 II as previously described [6]. Samples with a PMR value $>10$ were considered positive for methylation; samples were defined as CIMP high or CIMP low when $\geq 6$ or $1-5$ out of 8 CIMP markers had a PMR $>10$, respectively. CIMP negative was defined as 0 marker had a PMR $>10$.

\section{MSI analysis}

For the CRC cell line panel, MSI status was obtained from publicly available data from the Sanger institute and confirmed using publications [31,59]. Only the MSI status for OUMS-23 was not available, for which we performed the analysis. For determination of MSI status of the primary cell cultures, we used a PCR-based MSI Analysis System, version 1.2 (Promega, Leiden, the Netherlands), according to the manufacturer's instructions. In short, the standard 5 microsatellite amplicons were analysed for shortening to determine the presence of microsatellite instability. Samples were considered MSI when more than two out of five markers were instable.

\section{Mutation analyses of primary cell cultures}

Mutations in BRAF and KRAS were determined by a snapshot assay as previously described [60], supplemented by mutations in HRAS and NRAS. For TP53 a separate PCR-sequencing reaction was performed, which assesses the mutation hotspots. Primers were previously described [6]. 


\section{Wnt target and miR200 methylation}

Bisulfite conversion of gDNA was done by EZ-DNA methylation Gold-Kit (Zymo, Irvine, CA, USA). Percentage of methylation was determined using the PyroMark PCR system (Qiagen, Hilden, Germany). Bisulfite converted DNA was amplified using PyroMark PCR according to the manufacturer's instructions and PyroMark Assay Design Software 2.0 (Qiagen, Hilden, Germany) was used for primer design. Pyrosequencing reactions were performed following the manufacturer's instructions. Average methylation of all analysed CpGs of a particular gene was depicted as the percentage of methylation. PCR and sequencing primers are listed in Supplementary Table S6.

Acknowledgements The authors would like to thank M.C. Lecca for her work on the identification of the cell lines (short tandem repeat (STR) profiling) that was a valuable contribution to this paper. This work was supported by AMC, NWO Zwaartekracht, Cancer Genomics Center, the Dutch Cancer Society (KWF 2012-5735, 2015-7587 and 10150), TRANSCAN 2/TACTIC, the Dutch Gastrointestinal and Liver disorder Foundation (MLDS FP13-07) and AIRC $5 \times 1000$ (9979).

\section{Compliance with ethical standards}

Competing interests The authors declare that they have no competing financial interests.

\section{References}

1. Dienstmann R, Vermeulen L, Guinney J, Kopetz S, Tejpar S, Tabernero J, et al. Consensus molecular subtypes and the evolution of precision medicine in colorectal cancer. Nat Rev Cancer. 2017;17:79-92.

2. De Roock W, De Vriendt V, Normanno N, Ciardiello F, Tejpar S. KRAS, BRAF, PIK3CA, and PTEN mutations: implications for targeted therapies in metastatic colorectal cancer. Lancet Oncol. 2011;12:594-603.

3. Fearon ER, Vogelstein B. A genetic model for colorectal tumorigenesis. Cell. 1990;61:759-67.

4. Dunican DS, McWilliam P, Tighe O, Parle-McDermott A, Croke DT. Gene expression differences between the microsatellite instability (MIN) and chromosomal instability (CIN) phenotypes in colorectal cancer revealed by high-density cDNA array hybridization. Oncogene. 2002;21:3253-7.

5. IJspeert JE, Medema JP, Dekker E. Colorectal neoplasia pathways: state of the art. Gastrointest Endosc Clin $N \mathrm{Am}$. 2015;25:169-82.

6. De Sousa EMF, Wang X, Jansen M, Fessler E, Trinh A, de Rooij LP, et al. Poor-prognosis colon cancer is defined by a molecularly distinct subtype and develops from serrated precursor lesions. Nat Med. 2013;19:614-18.

7. Marisa L, de Reynies A, Duval A, Selves J, Gaub MP, Vescovo L, et al. Gene expression classification of colon cancer into molecular subtypes: characterization, validation, and prognostic value. PLoS Med. 2013;10:e1001453.

8. Budinska E, Popovici V, Tejpar S, D'Ario G, Lapique N, Sikora $\mathrm{KO}$, et al. Gene expression patterns unveil a new level of molecular heterogeneity in colorectal cancer. $J$ Pathol. 2013;231:63-76.
9. Schlicker A, Beran G, Chresta CM, McWalter G, Pritchard A, Weston S, et al. Subtypes of primary colorectal tumors correlate with response to targeted treatment in colorectal cell lines. BMC Med Genomics. 2012;5:66.

10. Roepman P, Schlicker A, Tabernero J, Majewski I, Tian S, Moreno V, et al. Colorectal cancer intrinsic subtypes predict chemotherapy benefit, deficient mismatch repair and epithelialto-mesenchymal transition. Int J Cancer. 2014;134:552-62.

11. Perez-Villamil B, Romera-Lopez A, Hernandez-Prieto S, LopezCampos G, Calles A, Lopez-Asenjo JA, et al. Colon cancer molecular subtypes identified by expression profiling and associated to stroma, mucinous type and different clinical behavior. BMC Cancer. 2012;12:260.

12. Guinney J, Dienstmann R, Wang X, de Reynies A, Schlicker A, Soneson C, et al. The consensus molecular subtypes of colorectal cancer. Nat Med. 2015;21:1350-56.

13. Grothey A, Van Cutsem E, Sobrero A, Siena S, Falcone A, Ychou $\mathrm{M}$, et al. Regorafenib monotherapy for previously treated metastatic colorectal cancer (CORRECT): an international, multicentre, randomised, placebo-controlled, phase 3 trial. Lancet. 2013;381:303-12.

14. Li J, Qin S, Xu R, Yau TC, Ma B, Pan H, et al. Regorafenib plus best supportive care versus placebo plus best supportive care in Asian patients with previously treated metastatic colorectal cancer (CONCUR): a randomised, double-blind, placebo-controlled, phase 3 trial. Lancet Oncol. 2015;16:619-29.

15. Le DT, Uram JN, Wang H, Bartlett BR, Kemberling H, Eyring $\mathrm{AD}$, et al. PD-1 blockade in tumors with mismatch-repair deficiency. N Engl J Med. 2015;372:2509-20.

16. Garnett MJ, Edelman EJ, Heidorn SJ, Greenman CD, Dastur A, Lau KW, et al. Systematic identification of genomic markers of drug sensitivity in cancer cells. Nature. 2012;483:570-5.

17. Barretina J, Caponigro G, Stransky N, Venkatesan K, Margolin AA, Kim S, et al. The cancer cell line encyclopedia enables predictive modelling of anticancer drug sensitivity. Nature. 2012;483:603-7.

18. Bertotti A, Migliardi G, Galimi F, Sassi F, Torti D, Isella C, et al. A molecularly annotated platform of patient-derived xenografts ("xenopatients") identifies HER2 as an effective therapeutic target in cetuximab-resistant colorectal cancer. Cancer Discov. 2011;1:508-523.

19. DeRose YS, Wang G, Lin YC, Bernard PS, Buys SS, Ebbert MT, et al. Tumor grafts derived from women with breast cancer authentically reflect tumor pathology, growth, metastasis and disease outcomes. Nat Med. 2011;17:1514-20.

20. Byrne AT, Alferez DG, Amant F, Annibali D, Arribas J, Biankin $\mathrm{AV}$, et al. Interrogating open issues in cancer precision medicine with patient-derived xenografts. Nat Rev Cancer. 2017;17:254-268.

21. Schutte M, Risch T, Abdavi-Azar N, Boehnke K, Schumacher D, Keil M, et al. Molecular dissection of colorectal cancer in preclinical models identifies biomarkers predicting sensitivity to EGFR inhibitors. Nat Commun. 2017;8:14262.

22. van de Wetering M, Francies HE, Francis JM, Bounova G, Iorio $\mathrm{F}$, Pronk $\mathrm{A}$, et al. Prospective derivation of a living organoid biobank of colorectal cancer patients. Cell. 2015;161: 933-945.

23. Weeber F, van de Wetering M, Hoogstraat M, Dijkstra KK, Krijgsman O, Kuilman, T, et al. Preserved genetic diversity in organoids cultured from biopsies of human colorectal cancer metastases. Proc Natl Acad Sci USA. 112, 13308-11 (2015).

24. Fujii M, Shimokawa M, Date S, Takano A, Matano M, Nanki K, et al. A colorectal tumor organoid library demonstrates progressive loss of niche factor requirements during tumorigenesis. Cell Stem Cell. 2016;18:827-838. 
25. Sato T, Stange DE, Ferrante M, Vries RG, Van Es JH, Van den Brink S, et al. Long-term expansion of epithelial organoids from human colon, adenoma, adenocarcinoma, and Barrett's epithelium. Gastroenterology. 2011;141:1762-72.

26. Song N, Pogue-Geile KL, Gavin PG, Yothers G, Kim SR, Johnson NL, et al. Clinical outcome from oxaliplatin treatment in stage II/III colon cancer according to intrinsic subtypes: secondary analysis of NSABP C-07/NRG oncology randomized clinical trial. JAMA Oncol. 2016;2:1162-9.

27. Del Rio M, Mollevi C, Bibeau F, Vie N, Selves J, Emile JF, et al. Molecular subtypes of metastatic colorectal cancer are associated with patient response to irinotecan-based therapies. Eur J Cancer. 2017:76:68-75.

28. Mouradov D, Sloggett C, Jorissen RN, Love CG, Li S, Burgess $\mathrm{AW}$, et al. Colorectal cancer cell lines are representative models of the main molecular subtypes of primary cancer. Cancer Res. 2014;74:3238-47.

29. Calon A, Lonardo E, Berenguer-Llergo A, Espinet E, HernandoMomblona X, Iglesias M, et al. Stromal gene expression defines poor-prognosis subtypes in colorectal cancer. Nat Genet. 2015;47:320-9.

30. Isella C, Terrasi A, Bellomo SE, Petti C, Galatola G, Muratore A, et al. Stromal contribution to the colorectal cancer transcriptome. Nat Genet. 2015;47:312-9.

31. Medico E, Russo M, Picco G, Cancelliere C, Valtorta E, Corti G, et al. The molecular landscape of colorectal cancer cell lines unveils clinically actionable kinase targets. Nat Commun. 2015;6:7002.

32. Network TCGA. Comprehensive molecular characterization of human colon and rectal cancer. Nature. 2012;487:330-337.

33. de Sousa EMF, Colak S, Buikhuisen J, Koster J, Cameron K, de Jong $\mathrm{JH}$, et al. Methylation of cancer-stem-cell-associated Wnt target genes predicts poor prognosis in colorectal cancer patients. Cell Stem Cell. 2011;9:476-85.

34. Fessler E, Jansen M, De Sousa EMF, Zhao L, Prasetyanti PR, Rodermond $\mathrm{H}$, et al. A multidimensional network approach reveals microRNAs as determinants of the mesenchymal colorectal cancer subtype. Oncogene. 2016;35:6026-37.

35. Chou J, Fitzgibbon MP, Mortales CL, Towlerton AM, Upton MP, Yeung RS, et al. Phenotypic and transcriptional fidelity of patientderived colon cancer xenografts in immune-deficient mice. PLoS ONE. 2013;8:e79874.

36. Isella C, Brundu F, Bellomo SE, Galimi F, Zanella E, Porporato $\mathrm{R}$, et al. Selective analysis of cancer-cell intrinsic transcriptional traits defines novel clinically relevant subtypes of colorectal cancer. Nat Commun. 2017;8:15107.

37. Trinh A, Trumpi K, De Sousa EMF, Wang X, de Jong JH, Fessler E, et al. Practical and robust identification of molecular subtypes in colorectal cancer by immunohistochemistry. Clin Cancer Res. 2017;23:387-398.

38. Kandimalla R, Linnekamp JF, van Hooff S, Castells A, Llor X, Andreu M, et al. Methylation of WNT target genes AXIN2 and DKK1 as robust biomarkers for recurrence prediction in stage II colon cancer. Oncogenesis. 2017;6:e308.

39. Zhang SS, Han ZP, Jing YY, Tao SF, Li TJ, Wang H, et al. CD133(+)CXCR4(+) colon cancer cells exhibit metastatic potential and predict poor prognosis of patients. BMC Med. 2012;10:85.

40. Wilding JL, Bodmer WF. Cancer cell lines for drug discovery and development. Cancer Res. 2014;74:2377-84.

41. Johnson KR, Wang L, Miller MC 3rd, Willingham MC, Fan W. 5Fluorouracil interferes with paclitaxel cytotoxicity against human solid tumor cells. Clin Cancer Res. 1997;3:1739-45.

42. Todaro M, Alea MP, Di Stefano AB, Cammareri P, Vermeulen L, Iovino $\mathrm{F}$, et al. Colon cancer stem cells dictate tumor growth and resist cell death by production of interleukin-4. Cell Stem Cell. 2007;1:389-402.

43. Fessler E, Drost J, van Hooff SR, Linnekamp JF, Wang X, Jansen $\mathrm{M}$, et al. TGFbeta signaling directs serrated adenomas to the mesenchymal colorectal cancer subtype. EMBO Molec Med. 2016;8:745-60.

44. Wagner KW, Punnoose EA, Januario T, Lawrence DA, Pitti RM, Lancaster K, et al. Death-receptor O-glycosylation controls tumorcell sensitivity to the proapoptotic ligand Apo2L/TRAIL. Nat Med. 2007;13:1070-77.

45. Arango D, Al-Obaidi S, Williams DS, Dopeso H, Mazzolini R, Corner $\mathrm{G}$, et al. Villin expression is frequently lost in poorly differentiated colon cancer. Am J Pathol. 2012;180:150921.

46. McCall MN, Bolstad BM, Irizarry RA. Frozen robust multiarray analysis (fRMA). Biostatistics. 2010;11:242-53.

47. Johnson WE, Li C, Rabinovic A. Adjusting batch effects in microarray expression data using empirical Bayes methods. Biostatistics. 2007;8:118-27.

48. Du P, Kibbe WA, Lin SM. Lumi: a pipeline for processing Illumina microarray. Bioinformatics. 2008;24:1547-48.

49. Irizarry RA, Hobbs B, Collin F, Beazer-Barclay YD, Antonellis $\mathrm{KJ}$, Scherf U, et al. Exploration, normalization, and summaries of high density oligonucleotide array probe level data. Biostatistics. 2003;4:249-64.

50. Ritchie ME, Phipson B, Wu D, Hu Y, Law CW, Shi W, et al. Limma powers differential expression analyses for RNAsequencing and microarray studies. Nucleic Acids Res. 2015;43: e47.

51. Meyer D, Dimitriadou E, Kurt H, Weingessel A, Leisch F, Chang C, et al. Misc Functions of the Department of Statistics, Probability Theory Group, (Formerly: E1071), TU Wien, R Package. 2017.

52. Futreal PA, Coin L, Marshall M, Down T, Hubbard T, Wooster R, et al. A census of human cancer genes. Nat Rev Cancer. 2004:4:177-83.

53. Gonzalez-Perez A, Perez-Llamas C, Deu-Pons J, Tamborero D, Schroeder MP, Jene-Sanz A, et al. IntOGen-mutations identifies cancer drivers across tumor types. Nat Methods. 2013;10:1081-82.

54. Shihab HA, Gough J, Cooper DN, Day IN, Gaunt TR. Predicting the functional consequences of cancer-associated amino acid substitutions. Bioinformatics. 2013;29:1504-10.

55. Calon A, Espinet E, Palomo-Ponce S, Tauriello DV, Iglesias M, Cespedes MV, et al. Dependency of colorectal cancer on a TGFbeta-driven program in stromal cells for metastasis initiation. Cancer Cell. 2012;22:571-584.

56. Subramanian A, Tamayo P, Mootha VK, Mukherjee S, Ebert BL, Gillette MA, et al. Gene set enrichment analysis: a knowledge-based approach for interpreting genome-wide expression profiles. Proc Natl Acad Sci USA. 2005;102:15545-50.

57. Efron B, Tibshirani R. On testing the significance of sets of genes. Ann Appl Stat.107-29.

58. Ogino S, Kawasaki T, Kirkner GJ, Kraft P, Loda M, Fuchs CS, et al. Evaluation of markers for $\mathrm{CpG}$ island methylator phenotype (CIMP) in colorectal cancer by a large population-based sample. $J$ Molec Diagn. 2007;9:305-14.

59. Ahmed D, Eide PW, Eilertsen IA, Danielsen SA, Eknaes M, Hektoen M, et al. Epigenetic and genetic features of 24 colon cancer cell lines. Oncogenesis. 2013;2:e71.

60. Lurkin I, Stoehr R, Hurst CD, van Tilborg AA, Knowles MA, Hartmann A, et al. Two multiplex assays that simultaneously identify 22 possible mutation sites in the KRAS, BRAF, NRAS and PIK3CA genes. PLoS ONE. 2010; 5:e8802. 